physiological variables such as the patient's temperature, blood pressure and pulse at diagnosis of CDAD were recorded as were any haematological and biochemical investigations taken within $24 \mathrm{~h}$ of diagnosis.

Results Hospital admissions between 14 and 30 days ( $p=0.031$ ), increasing co-morbidities $(p=0.05)$, systolic blood pressure $<100 \mathrm{~mm} \mathrm{Hg}(\mathrm{p}=0.048)$ and heart rate greater than 100 beats per minute $(p=0.043)$ were significantly associated with increased 30day mortality. There was a clear trend of increased mortality for increasing length of stay (LOS) on the current admission before development of diarrhoea (If LOS greater than 7 days before onset, $p=0.07)$, temperature $<35^{\circ} \mathrm{C} \quad(p=0.07)$, recent discharge from hospital within 30 days $(p=0.106)$ and to a lesser degree, low albumin (albumin $<30 \mathrm{~g} / \mathrm{l} \mathrm{p}=0.221$ ) at the time of diagnosis, age $>85$ years $(p=0.947)$ and current PPI use $(p=0.224)$. From the data, a scoring system was derived whereby 1 point was given to the nonstatistically significant trends and 2 points to the statistically significant trends. If there were 1 to 3 co-morbidities, this was scored as 1 and greater than 3 co-morbidities scored as 2 . There was a maximum score of 14 . On applying this retrospectively to the existing database, 39 patients were identified where all the parameters in the scoring system were available. Of these, a score of 4 or less was associated with low risk of 30-day mortality ( $0 \%$ mortality in 13 patients). A score of 5 to 7 was associated with moderate risk of 30 -day mortality (33.3\% mortality in 15 patients) and a score of 8 or more to be associated with a high risk of 30-day mortality $72.7 \%$ mortality in 11 patients).

Conclusion A simple scoring system holds promise for defining those patients at greatest risk of 30-day mortality from CDAD in our population. However a note of caution should be exercised. Applying a scoring system retrospectively to a dataset from which information has been used to derive the score does not validate it and a prospective study is planned to validate the York scoring system.

Competing interests None declared.

\section{PWE-104 PREVALANCE AND SIGNIFICANCE OF SESSILE SERRATED LESIONS FROM A LARGE PROSPECTIVE UK SERIES IN THE SETTING OF BOWEL CANCER SCREENING PROGRAMIME}

doi:10.1136/gutjnl-2012-302514d.104

S Tholoor, ${ }^{*}$ P Basford, P Bhandari. Department of Gastroenterology, Portsmouth Hospitals NHS Trust, Portsmouth, UK

Introduction Screening colonoscopy has not had a big impact in reducing right sided cancer mortality and it is believed that this could partly be due to sessile serrated lesions (SSLs) which are thought to be the precursors of microsatellite adenocarcinomas. In the UK, people from the age of 60 to 75 with positive faecal occult blood undergo screening colonoscopy. We aim to evaluate the prevalence and significance of SSLs in our screening population.

Methods All colonoscopies performed between 2007 and 2011 were prospectively recorded on a screening database along with the histology. Pathology was reported as per standards laid out by the National screening programme by accredited Pathologists.

Results We analysed 1948 polyps from a total of 1576 patients. 28/ 1948 polyps in 25/1576 patients were found to have sessile serrated lesions. This amounted to $1.4 \%$ of the total polyps found. Out of 25 patients, 13 patients were men. The prevalence of sessile serrated lesions in all patients with polyps was $1.7 \% 17 / 28$ lesions. (60\%) of the lesions were smaller than $10 \mathrm{~mm} .17 / 28(60 \%)$ were in the right colon. 14/28 (50\%) had no dysplasia. 14/28 (50\%) had low grade dysplasia. None had high grade dysplasia or cancer. No interval cancer was found in a mean follow-up of 12 months. All 25 patients with sessile serrated lesions were also found to have conventional adenomas. 1-2 adenomas were found in $6 / 25$ patients, 3-4 adenomas in $6 / 25$ patients and $13 / 25$ patients had at least 5 adenomas.

Prevalance of dysplasia: SSLs with dysplasia: 14/28 (50\%)

-Low grade dysplasia: 14/14, High grade dysplasia/cancer: 0/14 SSLs with NO dysplasia: 14/28 (50\%).

Conclusion (1) Our study shows a low prevalence of $1.7 \%$ of sessile serrated lesions accounting to $<1.5 \%$ of the total number of polyps found in the setting of bowel cancer screening programme. (2) We did not find any high grade dysplasia or cancer in these patients. No interval cancer or synchronous cancers were found. (3) All patients with SSLs had conventional adenomas and the presence of SSLs had no effect on their surveillance plans. (4) Our study does not show any clinical impact of the sessile serrated lesions on patient outcomes. This questions the clinical relevance of SSLs.

Abstract PWE-104 Table 1

\begin{tabular}{ll}
\hline Site & No of lesions \\
\hline Right colon & $17(60 \%)$ \\
Left colon & $11(40 \%)$ \\
Upto $10 \mathrm{~mm}$ & $17(60 \%)$ \\
$>10 \mathrm{~mm}$ & $11(40 \%)$ \\
\hline
\end{tabular}

Competing interests None declared.

\section{REFERENCES}

1. Lash RH, Genta RM, Schuler CM. Sessile serrated adenomas: prevalence of dysplasia and carcinoma in 2139 patients. J Clin Pathol 2010;63:681-6.

2. Freeman HJ. Heterogeneity of colorectal adenomas, the serrated adenoma, and implications for screening and surveillance. World J Gastroenterol 2008;14:3461-3.

\section{PWE-105 CLINICAL EFFICACY OF THE COMBINATION OF PROPOFOL AND KETAMINE (KETOFOL) FOR DEEP SEDATION FOR COLONOSCOPY}

doi:10.1136/gutjnl-2012-302514d.105

S Amornyotin,* W Chalayonnawin, S Kongphlay. Department of Anesthesiology, Faculty of Medicine Siriraj Hospital, Mahidol University, Bangkok, Thailand

Introduction A combination of propofol and ketamine is usually used to achieve sedation and analgesia during colonoscopy. Few studies have compared their efficacy. The aim of this study was to compare and evaluate the clinical efficacy of the combination of propofol and ketamine (ketofol) vs propofol alone when each regimen is used as sedative agents for colonoscopy.

Methods 194 patients who underwent colonoscopy in 2 years, were randomly assigned to $\mathrm{PN}$ and $\mathrm{PK}$ groups. 97 patients in group $\mathrm{PN}$ received propofol and normal saline and 97 patients in group PK received propofol and ketamine for intravenous sedation (IVS). All patients were premedicated with $0.02-0.03 \mathrm{mg} / \mathrm{kg}$ of midazolam. The primary outcome variable was the successfully completed colonoscopic procedure. The secondary outcome variables were patient tolerance, discomfort during insertion, patient and endoscopist satisfaction, haemodynamic responses, as well as complications during and immediately after procedure. Immediately after the procedure, the endoscopist was asked to rate tolerability for the patient, discomfort during insertion and satisfaction. As well, a blinded member of the research team evaluated the patient satisfaction, procedural pain, recovery time and recovery score.

Results All endoscopies were completely successfully. Mean total dose of propofol in group PK and PN was $6.98(2.90) \mathrm{mg} / \mathrm{hg} / \mathrm{h}$ and 7.73 (3.45) $\mathrm{mg} / \mathrm{kg} / \mathrm{h}$, respectively $(\mathrm{p}=0.413)$. Mean total dose of 
ketamine in group PK was $1.49(0.61) \mathrm{mg} / \mathrm{hg} / \mathrm{h}$. There were no significant differences in patient tolerance, discomfort during insertion, patient and endoscopist satisfaction, haemodynamic responses, procedural pain, recovery time and recovery score. Overall, cardiovascular and respiratory adverse events were not significantly different between the two groups. These adverse events were transient and easily treated with no sequelae.

Conclusion IVS in both regimens provided effective and safe for colonoscopy. Adverse events were relatively high in both groups. However, these adverse events were mild and transient. No serious adverse events were observed.

Competing interests None declared.

\section{PWE-106 IS IT NECESSARY FOR ALL COLORECTAL CANCER PATIENTS WITH LIVER METASTASIS TO BE DISCUSSED AT A HEPATOBILIARY MDT?}

doi:10.1136/gutjnl-2012-302514d.106

${ }^{1} \mathrm{~S}$ Vasudevan, ${ }^{*}{ }^{1} \mathrm{~J}$ Wright, ${ }^{2} \mathrm{M}$ Rees, ${ }^{2} \mathrm{~B}$ Creswell, ${ }^{1} \mathrm{D}$ Stiff, ${ }^{1} \mathrm{~A}$ Wordley, ${ }^{1} \mathrm{R}$ Motson. ${ }^{1}$ Colorectal Surgery, Colchester University Hospital, Colchester, UK; ${ }^{2}$ Hepatobiliary Surgery, Pelican Cancer Foundation, Basingstoke, UK

Introduction Current guidelines suggest an opinion from a hepatobiliary MDT should be sought for all patients with colorectal cancer liver metastasis. This places a significant burden of work on the hepatobiliary MDT who are often the referral centre for many hospitals (Approximately 20 cases per month at our referral centre). This study was performed to see if our local colorectal MDT was able to make a correct decision regarding referral for consideration of liver resection by comparing its decision with the decision from two hepatobiliary surgeons from our referral network.

Methods CT scans from 38 patients found to have liver metastasis from colorectal cancer were anonymised and sent to two hepatobiliary surgeons in our cancer network (Pelican Centre, Basingstoke). They classified them into three categories; U-Unresectable, CChemo to downstage then consider resection and $\mathrm{R}-$ Resectable. The results were then compared with the opinion of our local colorectal MDT made prior to the referral to the hepatobiliary MDT. Results The two independent hepatobiliary surgeons agreed with each other on 35/38 (92\%) of the CT scans. Our CRC MDT agreed with the hepatobiliary surgeons in $36 / 38$ (95\%) of cases. Only 6/29 (21\%) patients deemed suitable by review of the CT scan by the hepatobiliary surgeons went on to have a liver resection due to a variety of disease and patient factors.

Conclusion Our results show that our local colorectal MDT was able to make an accurate assessment of the need for referral for consideration of liver resection in this group of patients. We question the need for all similar cases to be "automatically" discussed with a hepatobiliary MDT such as those patients with wide spread liver disease. It is clear from the fact that only $21 \%$ of suitable patients for liver resection went on to have a resection that the colorectal MDT is making complex decisions based on many other patient factors.

Competing interests None declared.

\section{PWE-107 POTENTIAL ROLE OF INTRAVENOUS IMMUNOGLOBULIN TREATMENT IN THE MANAGEMENT OF PATIENTS WITH SEVERE AND RECURRENT CLOSTRIDIUM DIFFICILE INFECTION}

doi:10.1136/gutjnl-2012-302514d.107

${ }^{1,2} \mathrm{~T}$ M Monaghan, ${ }^{*}{ }^{1} \mathrm{M}$ Wilson, ${ }^{2} \mathrm{~K}$ Teahon, ${ }^{1,2}{ }^{2} \mathrm{R}$ Mahida. ${ }^{1}$ Institute of Infection, Immunity \& Inflammation, University of Nottingham, Nottingham, UK; ${ }^{2}$ Digestive
Diseases \& Thoracics Directorate, Nottingham University Hospitals NHS Trust, Nottingham, UK

Introduction Approximately $30 \%$ of patients with Clostridium difficile infection have recurrent disease, despite adequate treatment of the initial episode. Some patients with severe $C$ difficile-associated disease $(\mathrm{CDAD})$ fail to respond to standard treatment with oral vancomycin (125 $\mathrm{mg}$ three time a day). Intravenous immunoglobulin (IVIG) has been used in the management of these two groups of patients but no controlled trials have been reported and published clinical experience is mixed. We report our experience of the use of IVIG in these patients, including subsequent course of disease.

Methods Clinical information was collected prospectively and/or retrospectively (via review of case notes and hospital information system) in seven patients [median age 77 yrs (range 52-91 yrs), six female] with recurrent $\mathrm{CDAD}$ and five patients [62 (21-75) yrs, four female] with severe $C$ difficile-associated colitis. IVIG was used at a dose of $400 \mathrm{mg} / \mathrm{kg}$, rifaximin was given for 2 weeks (200 mg twice daily) and Saccharomyces boulardii for 4 weeks (125 mg four times a day).

Results Recurrent CDAD: median 5 (range 3-8) episodes of disease occurred over $9(6-15)$ months. Two patients (4 and 7 episodes) were treated with courses of vancomycin and did not subsequently have a recurrence over the following 5 and 40 months (respectively). In addition to vancomycin courses, three patients received rifaximin (two also had $S$ boulardii) and were free of recurrence over subsequent $15-27$ months. One patient (8 episodes) failed to respond to the above treatment, but after IVIG, there has been no recurrence (20 months follow-up). By contrast, a patient with myeloma did not respond to three IVIG infusions. Severe CDAD: CT showed colitis in all, CRP-107 (58-366) mg/l, WBC-17.2 (4.8-39.7) $\times$ $10^{9} / 1$. In addition to IVIG, all also had high dose oral vancomycin (500 mg four times a day) and intravenous metronidazole. Four (out of five) responded and were subsequently discharged and one had recurrence (responded to oral vancomycin). One patient, whose IVIG was delayed by $48 \mathrm{~h}$ after it was recommended, required a colectomy.

Conclusion (1) Some patients with multiple recurrences of CDAD may not require treatment in addition to courses of vancomycin but others may respond to IVIG. (2) There may be a role for IVIG in the management of patients with severe acute CDAD. (3) Our experience may facilitate identification of clinical characteristics to enable recruitment of suitable patients, with recurrent or severe CDAD, in multi-centre clinical trials of IVIG.

Competing interests None declared.

\section{PWE-108 TOXIN A-SPECIFIC ANTIGEN-ACTIVATED AND MEMORY B CELLS IN THE CIRCULATION OF PATIENTS WITH CLOSTRIDIUM DIFFICILE INFECTION}

doi:10.1136/gutjnl-2012-302514d.108

T M Monaghan, ${ }^{*}$ A Robins, H F Sewell, Y R Mahida. Institute of Infection, Immunity $\&$ Inflammation, University of Nottingham, Nottingham, UK

Introduction In Clostridium difficile infection, antibody-mediated immune response to secreted toxins $\mathrm{A}$ and $\mathrm{B}$ (which are also the main virulence factors) appears to be important in determining the nature of clinical disease. During a bacterial infection, activation of $\mathrm{B}$ cells leads to loss of immunoglobulin (Ig) D and expression of antigen-specific Ig on the cell surface. Following resolution of infection, antigen-specific memory B cells may be detectable in the circulation. Our aim was to identify circulating toxin A-activated B cells during clinical disease and toxin A-specific memory B cells following resolution of $C$ difficile infection. 